\title{
Estimating File-Spread in Delay Tolerant Networks under Two-Hop Routing
}

\author{
Arshad Ali ${ }^{1}$, Eitan Altman ${ }^{2}$, Tijani Chahed ${ }^{1}$, Dieter Fiems ${ }^{3}$, \\ Manoj Panda ${ }^{1, \star, \star \star}$, and Lucile Sassatelli ${ }^{4}$ \\ 1 Telecom SudParis, 9 rue C. Fourier, 91011 Evry Cedex, France \\ \{arshad.ali,tijani.chahed, manoj . panda\}@it-sudparis.eu \\ 2 INRIA, 2004 Route des Lucioles, 06902 Sophia-Antipolis, France \\ \{eitan.altman\}@sophia.inria.fr \\ 3 SMACS Research Group, \\ Department of Telecommunications and Information Processing (TW07), \\ St-Pietersnieuwstraat 41, B-9000 Gent, Belgium \\ dieter.fiems@ugent. be \\ 4 Laboratoire I3S, Université Nice Sophia Antipolis - CNRS, France \\ sassatelli@i3s.unice.fr
}

\begin{abstract}
We consider a Delay/Disruption Tolerant Network under two-hop routing. Our objective is to estimate and track the degree of spread of a message/file in the network. Indeed, having such real-time information is critical for on-line control of routing and energy expenditure. It also benefits the multi-casting application. With exponential inter-meeting times of mobile nodes: (i) for the estimation problem, we obtain exact expressions for the minimum mean-squared error (MMSE) estimator, and (ii) for the tracking problem, we first derive the diffusion approximations for the system dynamics and the measurements and then apply Kalman filtering. We also apply the solutions of the estimation and filtering problems to predict the time when a certain pre-defined fraction of nodes have received a copy of the message/file. Our analytical results are corroborated with extensive simulation results.
\end{abstract}

Keywords: delay/disruption tolerant networks; two-hop routing, multicasting, estimation and tracking, Kalman filtering, level-crossing.

\section{Introduction}

Sparse Mobile Ad hoc Networks (MANETs) are often referred to as Delay Tolerant Networks (DTNs) wherein a source has to rely on the mobility of other nodes which act as relays, and takes advantage of the transmission opportunities which occur when the mobile relays come into contact. This forwarding strategy is known as opportunistic routing. Several methods of spreading multiple copies of the same message (or packet or file) have been investigated under opportunistic routing [11, [10], [5. In epidemic routing [11] data packets are flooded to all

\footnotetext{
* Corresponding author.

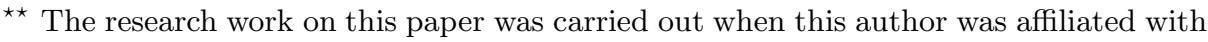
INRIA, Sophia Antipolis.
} 
nodes in the network to minimize the delay. In two-hop routing the relays do not give copies of the message to other relays.

As multiple copies of the same packet are allowed to spread in the network, it is important to track the number of copies so as to have an on-line adaptive replication policy. In this paper, we address this problem for the case of twohop routing. We assume that an observer node moves around in the network meeting with relay nodes to count the number of copies of the message. The nodes it meets inform it of whether they are carrying a copy of the packet. The problem is to get as accurate an estimation as possible of the number of nodes with copies using the measurements of the observer. The problem of adaptively controlling the spreading process will be addressed in the future.

Our Contributions: In this paper, under the assumption of exponential intermeeting times, we solve three problems. First, given the observer's count at time $t$, we derive the exact expressions for the instantaneous linear Minimum-MeanSquared-Error (MMSE) estimator. Second, we derive a discrete time Kalman filter based on diffusion approximations for the spreading process and the measurements. Third, we estimate/predict the time at which a certain given fraction of population has received copies of the file. All analyses are substantiated by discrete event simulations.

Comparison with Related Work: Mean-field approximations have been used to estimate the mean number of infected nodes under various spreading policies 13. Such approximations are accurate when the number of nodes is sufficiently large. Our approach of using measurements with MMSE estimator and Kalman filter allows us to track the discrepancy between the mean-field approximations and the actual sample path of the process. Furthermore, our Kalman filter estimation is based on a second-order approximation whereas the mean-field approximations are only first-order descriptions.

A related estimation problem in wire-line networks has been considered in [1, where the number of participants to a multicast session is tracked over time thanks to measurements taken by polling the users. In [1, the authors assume an infinite population from which arrivals occur and apply the diffusion approximation of the well-known $M / M / \infty$ queueing model. We, however, consider a realistic finite population from which arrivals occur. In [1], the Kalman filter is developed to track the fluctuations in the stationary regime of the $M / M / \infty$ queue. We, however, track the fluctuations in the transient phase. Furthermore, in [1, the delay in measurements (i.e., of the poll messages and the returning acknowledgments) is ignored. We, however, explicitly characterize the measurement process which complicates the derivation of the measurement equation.

\section{Network Model and Objectives}

We consider a Delay/Disruption Tolerant Network (DTN) consisting of $S_{0}$ sources and $N_{0}$ relay nodes. We focus on tracking the spread of one given file (or message) generated by these $S_{0}$ sources; tracking of other files generated by the same 
or other sources follows the same lines. The inter-meeting times of any specific (say, the $i$-th) source and any specific (say, the $j$-th) relay node are independent and exponentially distributed random variables with parameter $\beta$. At time zero, the $S_{0}$ sources start spreading a file adopting two-hop routing. Each time a source meets with a relay which does not already have a copy of the file, the relay gets a copy of the file. Recall that, in two-hop routing, the relays do not give copies of the file to other relays.

An observer $H$ monitors the system. The observer may be one of the sources, but not necessarily so. The inter-meeting times of the observer with any specific (say, the $k$-th) relay are independent and exponentially distributed random variables with parameter $\mu$. At each contact with a relay, the observer gets to know if the relay has or does not have a copy of the file.

Let $X(t)$ denote the number of relays that have a copy of the file at time t. Note that $X(t)$ does not include the sources. Let $Y(t)$ denote the number of copies that the observer has counted up to time $t$. Henceforth, we shall refer to $\{X(t), t \geq 0\}$ as "the process" and to $\{Y(t), t \geq 0\}$ as "the observation" or "the measurement". We assume that $X(0)=0$ and $Y(0)=0$.

Our objectives in this paper are to solve the following problems:

P1 Estimate the (value of the) process at time $t, X(t)$, given the observation at time $t, Y(t)$.

P2 Estimate the (value of the) process at time $t, X(t)$, given the history of observation, $\{Y(u), u \in U, U \subseteq[0, t]\}$.

P3 Estimate the time at which the process crosses a certain level $X_{L}$.

Problem P3 is motivated by multicast where one would be interested to know the time when a certain number, $X_{L}$, of nodes have received a copy of the file. Problems P1 and P2, as we shall see, can be seen as intermediate steps for solving Problem P3. But, they are also important problems in their own rights.

Our approach is to use linear estimators because they are simple to implement and useful in practice. For solving Problem P1, we use the linear MinimumMean-Squared-Error (MMSE) estimator, and for solving Problem P2, we use the Kalman filter. The Kalman filter is known to be optimal in several important ways [8, 9]. We solve Problem P3 using the solutions of Problems P1 and P2.

Problem P1: Consider two correlated random variables $X$ and $Y$, with their mean vector and covariance matrix given by $\left(\begin{array}{c}m_{x} \\ m_{y}\end{array}\right)$ and $\left(\begin{array}{cc}V_{x x} & V_{x y} \\ V_{y x} & V_{y y}\end{array}\right)$, respectively. We solve the optimal estimation problem P1 by applying Proposition 1

Proposition 1. The linear estimator of $X$ given $Y$ which minimizes the expected square estimation error is given by

$$
E[X \mid Y]=m_{x}+V_{x y} V_{y y}^{-1}\left(Y-m_{y}\right) .
$$

We derive the required means and covariances in Sect. 3.2

Problem P2: We approximate the process $\{X(t), t \geq 0\}$ by a diffusion process (Sect. 3.3). Sampling the approximate process at regular monitoring intervals 
of duration $T$, we obtain a discrete time linear stochastic difference equation for the process $\{X(t), t \geq 0\}$. We also derive a discrete time linear stochastic equation relating the measurements to the process. The linearity of both the system dynamics and the measurement equations allows us to apply the Kalman filter to use the previous estimation in order to update the current estimation optimally. The Kalman filter equations are derived in Sect. 3.4.

Problem P3: A first-order solution to the level-crossing problem is obtained by using the solution of Problem P1. A more accurate second-order solution is obtained by using the solution of Problem P2. The level-crossing analysis is carried out at the end of Sect. 4 .

\section{Dynamics of the File Spread and Observation}

In this section, guided by Proposition 1, we first derive the quantities $m_{x}, m_{y}$, $V_{x x}$, and $V_{x y}$ as functions of time. Then, we derive diffusion approximations for the process and the observation, and derive the corresponding discrete time linear stochastic equations by sampling at regular intervals.

\subsection{Characterization of the Process and Observation}

Let $T_{\lambda}^{i}$ denote the time at which relay $i$ receives a copy of the file. Note that $T_{\lambda}^{i}$ is exponentially distributed with parameter $\lambda=S_{0} \beta$. Then, the probability $p(t)$ that a relay has a copy of the file at time $t$ is given by

$$
p(t)=P\left(T_{\lambda}^{i} \leq t\right)=1-\exp (-\lambda t) .
$$

Let $\xi_{i}(t)$ denote the indicator variable that takes the value 1 if relay $i, i=$ $1, \ldots, N_{0}$, has a copy of the file at time $t$, and 0 otherwise. Then, we have

$$
X(t)=\sum_{i=1}^{N_{0}} \xi_{i}(t)
$$

Clearly, for each $i, i=1, \ldots, N_{0}$, and for each $t \geq 0, \xi_{i}(t)$ is a Bernoulli random variable with $P\left(\xi_{i}(t)=1\right)=p(t)$ and $P\left(\xi_{i}(t)=0\right)=1-p(t)$. By independence of source-relay meeting events, for each $t \geq 0$, the random variables $\xi_{i}(t), i=$ $1, \ldots, N_{0}$, are i.i.d., and we conclude that $X(t)$ has a Binomial distribution with parameters $N_{0}$ and $p(t)$, i.e.,

$$
P(X(t)=k)=\left(\begin{array}{c}
N_{0} \\
k
\end{array}\right) p(t)^{k}(1-p(t))^{N_{0}-k} .
$$

Given the process $\{X(t), t \geq 0\}$, the measurement, $\{Y(t), t \geq 0\}$, is a doublystochastic Poisson process [2] with (stochastic) intensity function $\mu X(t)$. Thus, the count of the observer, $Y(t)$, has a Poisson distribution with parameter

$$
\theta_{y}(t)=\mu \int_{0}^{t} X(u) d u
$$

We emphasize that, $\forall t \geq 0, \theta_{y}(t)$ is a random variable, since $X(t)$ is stochastic. 


\subsection{Derivation of the Means and (Co)variances}

Lemma 1. (i) $X(t)$ has mean $m_{x}(t)$ and variance $V_{x x}(t)$ given by

$$
m_{x}(t)=N_{0} p(t) \quad, \quad V_{x x}(t)=N_{0} p(t)(1-p(t))=m_{x}(t)(1-p(t)) .
$$

(ii) $Y(t)$ has mean $m_{y}(t)$ and variance $V_{y y}(t)$ given by

$$
m_{y}(t)=\mu m_{x}(t) E\left[T_{x}(t)\right]
$$

and

$$
V_{y y}(t)=m_{y}(t)+m_{x}(t) \mu^{2}\left(E\left[T_{x}^{2}(t)\right]-\left(E\left[T_{x}(t)\right]\right)^{2}\right)+\mu^{2} E\left[T_{x}^{2}(t)\right] V_{x x}(t),
$$

where

$E\left[T_{x}(t)\right]=\frac{t}{1-\exp (-\lambda t)}-\frac{1}{\lambda} \quad, \quad E\left[T_{x}^{2}(t)\right]=\frac{\exp (\lambda t)}{\lambda^{3}}\left(\lambda^{2} t^{2}-2 \lambda t+2\right)-\frac{2}{\lambda^{3}}$.

(iii) The covariance between $X(t)$ and $Y(t)$ is given by

$$
V_{y x}(t)=m_{y}(t)(1-p(t)) .
$$

Proof. (i) Follows directly from (3).

(ii) Substituting (2) in (4), and interchanging the order of summation and integration, we obtain

$$
\theta_{y}(t)=\sum_{i=1}^{N_{0}} \mu \int_{0}^{t} \xi_{i}(u) d u=\sum_{i=1}^{N_{0}} \mu \max \left(t-T_{\lambda}^{i}, 0\right)=\sum_{i=1}^{X(t)} \mu T_{x}^{i}(t),
$$

where $T_{x}^{i}(t)$ are i.i.d. random variables, each distributed like the truncated random variable $T_{x}(t)$ (truncated at $t$ ) with the following distribution:

$$
P\left(T_{x}(t)>a\right)=P\left(t-T_{\lambda}^{i}>a \mid T_{\lambda}^{i} \leq t\right)=\frac{1-\exp (-\lambda(t-a))}{1-\exp (-\lambda t)} \quad \text { for } 0 \leq a \leq t .
$$

$E\left[T_{x}(t)\right]$ and $E\left[T_{x}^{2}(t)\right]$ as given above follow from (5). Then,

$$
\begin{aligned}
m_{y}(t) & =E[Y(t)]=E_{X}\left[E_{Y}[Y(t) \mid X(t)]\right]=E\left[\theta_{y}(t)\right]=\mu m_{x}(t) E\left[T_{x}(t)\right] . \\
V_{y y}(t) & =\operatorname{Var}(Y(t))=E_{X}\left[\operatorname{Var}_{Y}(Y(t) \mid X(t))\right]+\operatorname{Var}_{X}\left(E_{Y}[Y(t) \mid X(t)]\right) \\
& =E\left[\theta_{y}(t)\right]+\operatorname{Var}\left(\theta_{y}(t)\right),
\end{aligned}
$$

since the variance of a Poisson random variable is equal to its mean. As before, $E\left[\theta_{y}(t)\right]=m_{y}(t)$ and $\operatorname{Var}\left(\theta_{y}(t)\right)$ is obtained as follows:

$$
\begin{aligned}
\operatorname{Var}\left(\theta_{y}(t)\right) & =E[X(t)] \operatorname{Var}\left(\mu T_{x}(t)\right)+E\left[\mu^{2} T_{x}(t)^{2}\right] \operatorname{Var}(X(t)) \\
& =m_{x}(t) \mu^{2}\left(E\left[T_{x}^{2}(t)\right]-\left(E\left[T_{x}(t)\right]\right)^{2}\right)+\mu^{2} E\left[T_{x}^{2}(t)\right] V_{x x}(t) .
\end{aligned}
$$

(iii) $V_{y x}(t)=V_{x y}(t)=E[X(t) Y(t)]-E[X(t)] E[Y(t)]$

$$
\begin{aligned}
& =E_{X}\left[E_{Y}[X(t) Y(t) \mid X(t)]\right]-m_{x}(t) m_{y}(t) \\
& =E\left[X(t) \theta_{y}(t)\right]-m_{x}(t) m_{y}(t)=E\left[X^{2}(t)\right] \mu E\left[T_{x}(t)\right]-m_{x}(t) m_{y}(t) \\
& =\left(V_{x x}(t)+\left(m_{x}(t)\right)^{2}\right) \mu E\left[T_{x}(t)\right]-m_{x}(t) m_{y}(t)=m_{y}(t)(1-p(t))(9)
\end{aligned}
$$




\subsection{Fluid and Diffusion Approximations}

The process $\{X(t), t \geq 0\}$ can be viewed either as a state-dependent queue [7] or as a density-dependent Markov process [4. We obtain the fluid and diffusion approximations for the process $\{X(t), t \geq 0\}$ by viewing it as a single-server Markovian queue with state-dependent arrival rates, zero service rate and infinite buffer, and then applying the framework of [7. A brief informal background on fluid and diffusion approximations has been provided in the Appendix.

Consider the sequence $M_{X}^{(n)} / M_{X}^{(n)} / 1 / \infty / n, n=1,2, \ldots$, of state-dependent Markovian queueing systems, where the index $n$ denotes the size of the population from which the arrivals are drawn and $X^{(n)}(t)$ denotes the queue length at time $t$ of the $n$-th system. The analogy with our DTN is as follows. The quantities $n$ and $X^{(n)}(t)$ of the queueing system correspond to the quantities $N_{0}$ and $X(t)$, respectively, in our DTN. In analogy with our DTN, we let the arrival and departure rates for the $n$-th queueing system at state $X^{(n)}$ to be

$$
\lambda^{(n)}\left(X^{(n)}\right)=\lambda\left(n-X^{(n)}\right), \quad \text { and } \quad \mu^{(n)}\left(X^{(n)}\right)=0,
$$

respectively. Note that, as the number of relays with a copy, $X^{(n)}$, increases, the rate of increase, $\lambda^{(n)}$, (of $X^{(n)}$ ) decreases because the number of relays not having a copy, $n-X^{(n)}$, decreases. Also, the rate of decrease, $\mu^{(n)}$, (of $\left.X^{(n)}\right)$ is zero because $X^{(n)}$ never decreases.

Consider the sequence of processes $\left\{Y^{(n)}(t), t \geq 0\right\}, n=1,2, \ldots$, where, for each $n,\left\{Y^{(n)}(t), t \geq 0\right\}$ is a doubly stochastic Poisson process with stochastic intensity function $\mu X^{(n)}(t)$, i.e., we have

$$
Y^{(n)}(t)=\mathcal{P}\left(\mu \int_{0}^{t} X^{(n)}(u) d u\right),
$$

where $\{\mathcal{P}(t), t \geq 0\}$ denotes a Poisson process of unit intensity. Clearly, for each $n, Y^{(n)}(t)$ denotes the observer count at time $t$ corresponding to the $n$-th system. First, we obtain the fluid limits of the process and the measurement.

Lemma 2. (i) Let $x^{(n)}(t):=X^{(n)}(t) / n$. The fluid limit $\{x(t), t \geq 0\}$ associated with the sequence $\left\{X^{(n)}(t), t \geq 0\right\}, n=1,2, \ldots$, i.e., the limit of the sequence $\left\{x^{(n)}(t), t \geq 0\right\}, n=1,2, \ldots$, as $n \uparrow \infty$, is given by

$$
x(t)=1-\exp (-\lambda t) \text {, where } \lambda=S_{0} \beta .
$$

(ii) Let $y^{(n)}(t):=Y^{(n)}(t) / n$. The fluid limit $\{y(t), t \geq 0\}$ associated with the sequence $\left\{Y^{(n)}(t), t \geq 0\right\}, n=1,2, \ldots$, i.e., the limit of the sequence $\left\{y^{(n)}(t), t \geq\right.$ $0\}, n=1,2, \ldots$, as $n \uparrow \infty$, is given by

$$
y(t)=\mu \int_{0}^{t} x(u) d u .
$$

Proof. (i) Applying Theorem 4.1 of [7] (or, Theorem 3.1 of [4]), the fluid limit $\{x(t), t \geq 0\}$ associated with the sequence $\left\{X^{(n)}(t), t \geq 0\right\}, n=1,2, \ldots$, is given 
by the unique solution to the Ordinary Differential Equation (ODE) $\frac{d x(t)}{d t}=$ $\lambda(1-x(t))$, with initial condition $x(0)=0$, where $\lambda=S_{0} \beta$, whereby the result. (ii) Consider the mappings $\phi_{1, n}(t)=\frac{\mathcal{P}(n t)-n t}{n}$, and $\phi_{2, n}(t)=\frac{\mu}{n} \int_{0}^{t} X^{(n)}(u) d u=$ $\mu \int_{0}^{t} x^{(n)}(u) d u$. It is easy to see that $y^{(n)}(t)=\left(\phi_{1, n} \circ \phi_{2, n}\right)(t)+\phi_{2, n}(t)$, where $(f \circ$ $g)(x)$ is the composition $f(g(x))$. As $n \uparrow \infty$, we have $\phi_{1, n}(t) \rightarrow 0$ and $\phi_{2, n}(t) \rightarrow$ $\mu \int_{0}^{t} x(u) d u$, almost surely. Applying the Continuous Mapping Theorem (CMT) (see Theorem 13.2.1 of [12]), we obtain the fluid limit $\{y(t), t \geq 0\}$.

Next, we obtain the diffusion limits of the process and the measurement.

Theorem 1. (i) Let $v_{x}^{(n)}(t):=\sqrt{n}\left(x^{(n)}(t)-x(t)\right)$. The diffusion limit $\left\{v_{x}(t), t \geq\right.$ $0\}$ associated with the sequence $\left\{X^{(n)}(t), t \geq 0\right\}, n=1,2, \ldots$, i.e., the limit of the sequence $\left\{v_{x}^{(n)}(t), t \geq 0\right\}, n=1,2, \ldots$, as $n \uparrow \infty$, is given by

$$
v_{x}(t)=\sqrt{\lambda} \int_{0}^{t} e^{-\lambda(t-u / 2)} d B_{1}(u),
$$

where $B_{1}(t)$ is a standard Brownian motion.

(ii) Let $v_{y}^{(n)}(t):=\sqrt{n}\left(y^{(n)}(t)-y(t)\right)$. The diffusion limit $\left\{v_{y}(t), t \geq 0\right\}$ associated with the sequence $\left\{Y^{(n)}(t), t \geq 0\right\}, n=1,2, \ldots$, i.e., the limit of the sequence $\left\{v_{y}^{(n)}(t), t \geq 0\right\}, n=1,2, \ldots$, as $n \uparrow \infty$, is given by

$$
v_{y}(t)=\int_{0}^{t} \sqrt{\mu x(u)} d B_{2}(u)+\frac{\mu}{\sqrt{\lambda}} \int_{0}^{t} e^{-\lambda u / 2} d B_{1}(u)-\frac{\mu}{\lambda} v_{x}(t),
$$

where $B_{2}(t)$ is a standard Brownian motion independent of $B_{1}(t)$.

Proof. (i) Applying Theorem 4.2 of [7], the diffusion limit $\left\{v_{x}(t), t \geq 0\right\}$ associated with the sequence $\left\{X^{(n)}(t), t \geq 0\right\}, n=1,2, \ldots$, is given by the unique (strong) solution to the linear Stochastic Differential Equation (SDE)

$$
d v_{x}(t)=-\lambda v_{x}(t) d t+\sqrt{\lambda(1-x(t))} d B_{1}(t)
$$

with initial condition $v_{x}(0) \sim \mathcal{N}(0,0)$, where $B_{1}(t)$ denotes a standard Brownian motion. Solving (16) (see page 354 of [3]), we obtain the result for all $0 \leq t<\infty$. (ii) Defining the mapping $\phi_{3, n}(t)=\frac{\mathcal{P}(n t)-n t}{\sqrt{n}}$, it is easy to see that

$$
v_{y}^{(n)}(t)=\left(\phi_{3, n} \circ \phi_{2, n}\right)(t)+\mu \int_{0}^{t} v_{x}^{(n)}(u) d u .
$$

Noting that the diffusion limit associated with $\phi_{3, n}(t)$ is a standard Brownian motion $B_{2}(t)$ (which is independent of $B_{1}(t)$ on which $v_{x}(t)$ depends), and applying CMT (see Theorem 13.2.1 of [12]), we obtain the diffusion limit $\left\{v_{y}(t), t \geq 0\right\}$ associated with the sequence $\left\{Y^{(n)}(t), t \geq 0\right\}, n=1,2, \ldots$, as

$$
v_{y}(t)=B_{2}\left(\mu \int_{0}^{t} x(u) d u\right)+\mu \int_{0}^{t} v_{x}(u) d u .
$$

The result is obtained by simplifying the above equation. 


\subsection{The Kalman Filter}

Defining $v_{x, k}:=v_{x}(k T)$, where $T(>0)$ is some periodic interval at which we want to track the process $X(t)$, we obtain from (14) the system dynamic equation as:

$$
v_{x, k+1}=\alpha v_{x, k}+w_{k}, \quad k=0,1,2, \ldots,
$$

where $\alpha=e^{-\lambda T}$, and

$$
w_{k}=\sqrt{\lambda} \int_{k T}^{(k+1) T} e^{-\lambda((k+1) T-u / 2)} d B_{1}(u) .
$$

Defining $v_{y, k}:=v_{y}(k T)$, we obtain from (15) the measurement equation as:

$$
v_{y, k}=\gamma v_{x, k}+z_{k}, \quad k=0,1,2, \ldots,
$$

where $\gamma=-\frac{\mu}{\lambda}$, and $z_{k}=r_{k}+s_{k}$, where

$$
r_{k}=\frac{\mu}{\sqrt{\lambda}} \int_{0}^{k T} e^{-\lambda u / 2} d B_{1}(u), \quad \text { and } \quad s_{k}=\int_{0}^{k T} \sqrt{\mu x(u)} d B_{2}(u) .
$$

Defining, $n_{k}:=n_{1, k}+n_{2, k}$, where

$$
n_{1, k}=\frac{\mu}{\sqrt{\lambda}} \int_{k T}^{(k+1) T} e^{-\lambda u / 2} d B_{1}(u), \quad \text { and } \quad n_{2, k}=\int_{k T}^{(k+1) T} \sqrt{\mu x(u)} d B_{2}(u),
$$

we obtain, $r_{k+1}=r_{k}+n_{1, k}, s_{k+1}=s_{k}+n_{2, k}$ and $z_{k+1}=z_{k}+n_{k}$.

Notice that, the process noise $w$ is white, but the measurement noise $z$ is colored. We whiten the measurement noise by defining $v_{y, k}^{\prime}:=v_{y, k+1}-v_{y, k}$, and derive the modified measurement equation as:

$$
\begin{aligned}
v_{y, k}^{\prime} & =v_{y, k+1}-v_{y, k}=\gamma v_{x, k+1}+z_{k+1}-\gamma v_{x, k}-z_{k} \\
& =\gamma\left(\alpha v_{x, k}+w_{k}\right)+z_{k+1}-\gamma v_{x, k}-z_{k}=\gamma^{\prime} v_{x, k}+z_{k}^{\prime},
\end{aligned}
$$

where $\gamma^{\prime}=\gamma(\alpha-1)$ and $z_{k}^{\prime}=\gamma w_{k}+n_{k}$. Notice that the modified measurement noise $z^{\prime}$ is white. The modified measurement noise $z^{\prime}$ and the original (unmodified) measurement noise $z$ are both correlated with the process noise $w$. However, the modified measurement noise at (the discrete) time $k, z_{k}^{\prime}$, is uncorrelated with the process noise up to time $k-1,\left\{w_{j}\right\}, j=0,1, \ldots, k-1$. Thus, $M_{k}:=E\left[w_{k-1} z_{k}^{\prime}\right]=0$, and we can apply a standard Kalman filter (see page 187 of [9]) with the system dynamics and (modified) measurement equations

$$
\begin{aligned}
& v_{x, k}=\alpha v_{x, k-1}+w_{k-1}, \\
& v_{y, k}^{\prime}=\gamma^{\prime} v_{x, k}+z_{k}^{\prime},
\end{aligned}
$$

where $\left\{w_{k}, k=0,1, \ldots\right\}$ and $\left\{z_{k}^{\prime}, k=0,1, \ldots\right\}$ are white noise sequences with $w_{k} \sim \mathcal{N}\left(0, Q_{k}\right), \quad Q_{k}:=E\left[w_{k}^{2}\right], \quad$ and $\quad z_{k}^{\prime} \sim \mathcal{N}\left(0, R_{k}\right), \quad R_{k}:=E\left[\left(z_{k}^{\prime}\right)^{2}\right]$. 
It can be shown that

$$
\begin{gathered}
E\left[w_{k}^{2}\right]=(1-\alpha) \alpha^{k+1}, \text { and } E\left[\left(z_{k}^{\prime}\right)^{2}\right]=\gamma^{2} E\left[w_{k}^{2}\right]+2 \gamma E\left[w_{k} n_{k}\right]+E\left[n_{k}^{2}\right], \text { where } \\
E\left[w_{k} n_{k}\right]=\mu T \alpha^{k+1}, \quad \text { and } E\left[n_{k}^{2}\right]=\mu T-\gamma(1-\gamma)(1-\alpha) \alpha^{k} .
\end{gathered}
$$

Let $\hat{v}_{x, k}^{-}$and $\hat{v}_{x, k}^{+}$denote the estimates for $v_{x, k}$ before and after taking into account the measurement, respectively, at time $k$. Let $P_{k}^{-}$and $P_{k}^{+}$denote the covariances of the corresponding estimation errors. Let $\hat{v}_{y, k}^{\prime}=\hat{v}_{y, k+1}-\hat{v}_{y, k}$, where $\hat{v}_{y, k}=\sqrt{N_{0}}\left(\left(\hat{Y}(k T) / N_{0}\right)-y(k T)\right)$, and $\hat{Y}(k T)$ and $y(k T)$ denote the actual measurement (i.e., observer count) and the value of $y(t)$, respectively, at time $t=k T$. Starting with $\hat{v}_{x, 0}^{+}=0$ and $P_{0}^{+}=0$, we apply the following Kalman filter equations (see Equations 5.17-5.19 of [9]) repeatedly at all time $k$ :

$$
\begin{aligned}
\hat{v}_{x, k}^{-} & =\alpha \hat{v}_{x, k-1}^{+}, \\
P_{k}^{-} & =\alpha^{2} P_{k-1}^{+}+Q_{k-1}, \\
P_{k}^{+} & =\left(\left(P_{k}^{-}\right)^{-1}+\left(\gamma^{\prime}\right)^{2} / R_{k}\right)^{-1}, \\
K_{k} & =\gamma^{\prime} P_{k}^{+} / R_{k}, \\
\hat{v}_{x, k}^{+} & =\hat{v}_{x, k}^{-}+K_{k}\left(\hat{v}_{y, k}^{\prime}-\gamma^{\prime} \hat{v}_{x, k}^{-}\right),
\end{aligned}
$$

where $K_{k}$ denotes the Kalman filter gain at time $k$. We obtain the estimates for the process as $\hat{X}(k T)=N_{0} x(k T)+\sqrt{N_{0}} \hat{v}_{x, k}^{+}$, where $\sqrt{N_{0}} \hat{v}_{x, k}^{+}$provides an estimate of the fluctuation of the process about its mean, at time $t=k T$.

\section{Performance of Analytical Prediction and Estimation Based on Measurements}

In this section, we evaluate: (i) the quality of estimation provided by the MMSE estimator and the Kalman filter, and (ii) the accuracy of the predictions about the level-crossing times based on the estimation. We also comment on the prediction effectiveness of the fluid model of the process. We simulate a DTN as described in Sect. 2 for the following scenarios:

$\begin{array}{lllll}\text { Scenario 1: } & N_{0}=50, & \beta=0.02, & T=1.0, & \mu=\beta . \\ \text { Scenario 2: } & N_{0}=50, & \beta=0.02, & T=0.1, & \mu=10 \beta . \\ \text { Scenario 3: } & N_{0}=50, & \beta=0.02, & T=0.01, & \mu=100 \beta . \\ \text { Scenario 4: } & N_{0}=200, & \beta=0.02, & T=1.0, & \mu=\beta . \\ \text { Scenario 5: } & N_{0}=1000, & \beta=0.02, & T=1.0, & \mu=\beta .\end{array}$

In Fig. 11 we depict the performance of the MMSE estimator and the Kalman filter for Scenario 1. We note that the estimations by both the MMSE estimator and the Kalman filter are very close to each other and indeed close to the fluid approximation $N_{0} x(t)$ of $X(t)$. In Fig. 2, we show the estimations of the fluctuations about the fluid limit for Scenario 1, and notice that neither the MMSE estimator nor the Kalman filter is able to successfully track the fluctuations in 


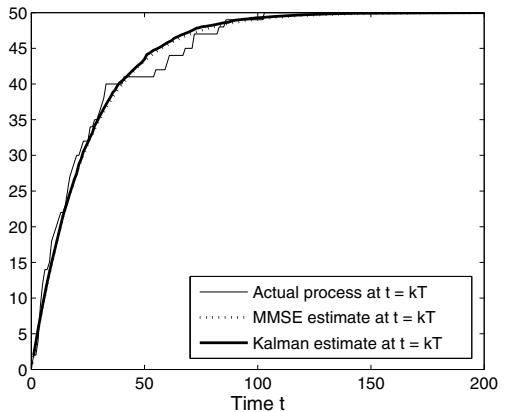

Fig. 1. Performance of MMSE estimation Fig. 2. MMSE estimation and Kalman filand Kalman filter estimation of the process ter estimation of the process fluctuations for Scenario 1

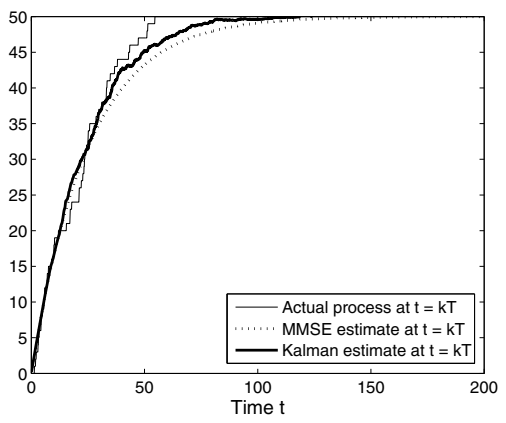

Fig. 3. Performance of MMSE estimation and Kalman filter estimation of the process for Scenario 2

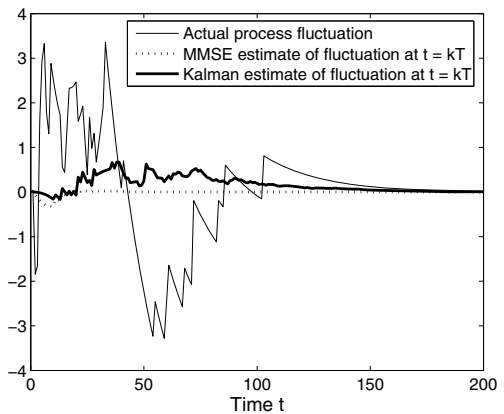

for Scenario 1

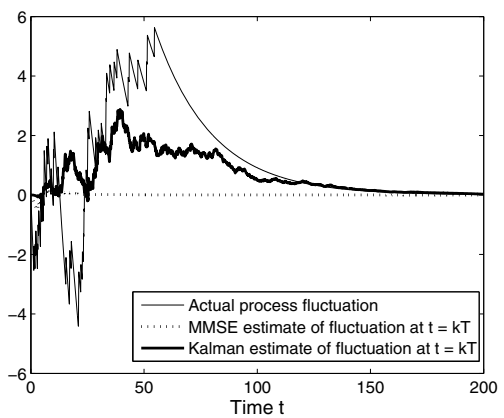

Fig. 4. MMSE estimation and Kalman filter estimation of the process fluctuations for Scenario 2

this scenario. We suspect that the inability to track the fluctuations in Scenario 1 is primarily due to the insufficiency of measurement data.

To verify if the inability to track the fluctuations in Scenario 1 is indeed due to the insufficiency of measurement data, we examine Scenario 2 (Figs. 3 and 4) and Scenario 3 (Figs. 5 and 6). In Scenario 2 (resp. Scenario 3), we increase the rate $\mu$ at which measurements are taken by the observer by a factor 10 (resp. 100). To make better use of faster measurements and avoid smoothing of measurement data over longer time intervals, we also decrease the monitoring interval $T$ by the same factor. We observe that the performance of the Kalman filter is much improved in Scenario 2 with faster measurements during the later phase of spreading (compare Fig. 4 with Fig. 2). Comparing Fig. 6 with Figs. 4 and 2 . we observe that tracking of the fluctuations by the Kalman filter is extremely accurate in Scenario 3. This accurate tracking of the fluctuations results in extremely accurate tracking of the process itself (see Fig. 5). 


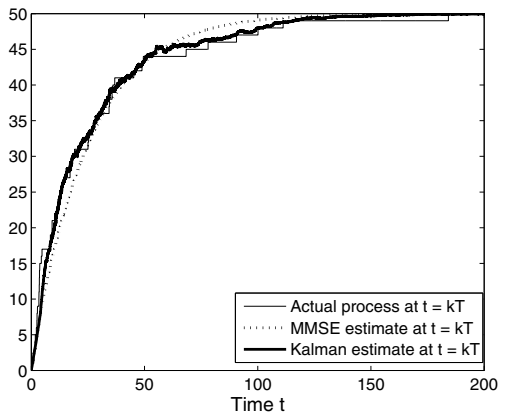

Fig. 5. Performance of MMSE estimation Fig. 6. MMSE estimation and Kalman filand Kalman filter estimation of the process ter estimation of the process fluctuations for Scenario 3

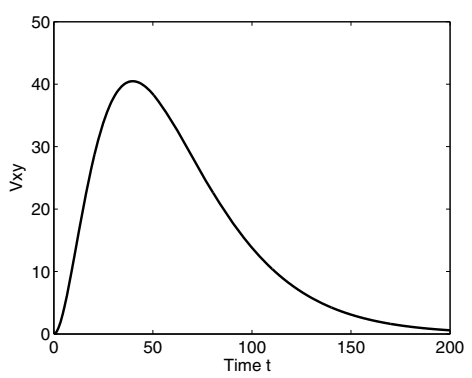

Fig. 7. $V_{x y}(t)$ as a function of time

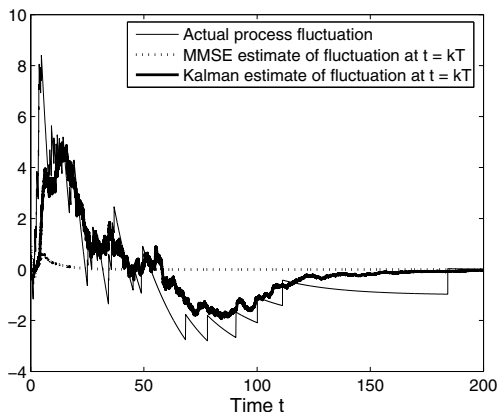

for Scenario 3

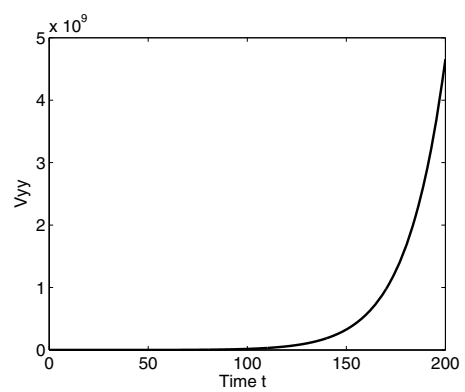

Fig. 8. $V_{y y}(t)$ as a function of time

In Figs. 3, 6, we observe that the MMSE estimator fails to make use of faster measurements. In fact, it stays very close to the fluid approximation. This can be explained as follows. The MMSE estimation differs from the fluid approximation $N_{0} x(t)$ (which is equal to $\left.m_{x}(t)\right)$ by the term $V_{x y}(t) V_{y y}(t)^{-1}\left(Y(t)-m_{y}(t)\right.$ ). From the expressions for $V_{x y}(t)$ and $V_{y y}(t)$ in Sect. 3.2, it can be seen that $V_{x y}(t)$ initially increases sub-linearly, but then quickly decreases exponentially with $t$ (see Fig. 7), and $V_{y y}(t)$ increases super-linearly with $t$ (see Fig. 8). Thus, except for an initial phase, the effect of the measurement $\left(Y(t)-m_{y}(t)\right)$ is diminished by the factor $V_{x y}(t) V_{y y}(t)^{-1}$. Increasing $\mu$ by a factor $K$ increases $V_{x y}(t)$ by a factor $K$, but also increases $V_{y y}(t)$ by a factor $K^{2}$. Thus, increasing $\mu$ by a factor $K$ results in an overall attenuation of the measurement $\left(Y(t)-m_{y}(t)\right)$ by a factor $K$ (see Sect. 3.2). Furthermore, the difference between the measurement $Y(t)$ and its mean $m_{y}(t)$ also decreases with $t$. In summary, we can expect the performance of the MMSE estimator to get worse with time.

Next, we examine the situations in which the fluid approximation itself can be used as a good predictor. Suppose that we increase the area of the network by a factor $K$ keeping the density of nodes constant. Then, we increase both 


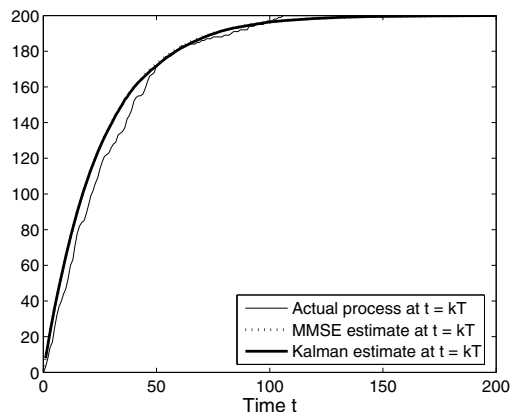

Fig. 9. Performance of MMSE estimation and Kalman filter estimation of the process with Scenario 4

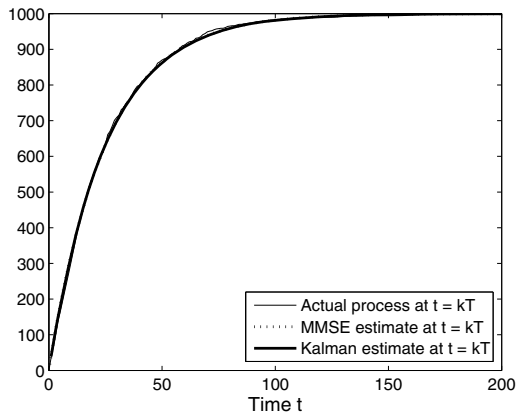

Fig. 10. Performance of MMSE estimation and Kalman filter estimation of the process with Scenario 5

$S_{0}$ and $N_{0}$ by $K$, but decrease the source-relay meeting rate $\beta$ by $K$. Then, the net rate at which meetings occur in the network increases from $S_{0} N_{0} \beta=\lambda N_{0}$ to $K S_{0} N_{0} \beta=\lambda\left(K N_{0}\right)$. This scaling is equivalent to increasing only $N_{0}$ by a factor $K$ keeping $S_{0}$ and $\beta$ constant as in (10). Thus, if the area of the network is large so that $N_{0}$ is large, then the fluid model can be a good predictor. We demonstrate this by Figs. 9 and 10 which correspond to Scenarios 4 and 5, respectively. Note that Scenarios 4 and 5 are derived from Scenario 1 by scaling as above with a scaling factor $K=4$ and $K=20$, respectively. Comparing Figs. 1, 9 and 10, we observe that the process becomes smoother and closer to the fluid approximation with increase in the number of nodes $N_{0}$.

Level-Crossing Times: Next, we compare the accuracy of the MMSE and the Kalman estimators in estimating the level-crossing times by computing the percentage error w.r.t. the level-crossing times of the actual process and averaging over 100 runs. Fixing the threshold levels at $X_{L}=0.15 N_{0}, 0.25 N_{0}, 0.50 N_{0}, 0.75 N_{0}$, and $0.90 N_{0}$, we obtained average percentage errors for estimates of level-crossing times by the MMSE and the Kalman estimators for Scenario 3. We summarize the results as follows:

$$
\begin{array}{llll}
X_{L}=0.15 N_{0}, & e(M M S E)=25.32 \%, & e(\text { Kalman })=23.72 \% \\
X_{L}=0.25 N_{0}, & e(M M S E)=22.63 \%, & e(\text { Kalman })=16.07 \% \\
X_{L}=0.50 N_{0}, & e(M M S E)=14.98 \%, & e(\text { Kalman })=8.32 \% \\
X_{L}=0.75 N_{0}, & e(M M S E)=12.71 \%, & e(\text { Kalman })=7.33 \% \\
X_{L}=0.90 N_{0}, & e(M M S E)=14.80 \%, & e(\text { Kalman })=9.94 \%
\end{array} .
$$

We conducted similar experiments (not reported here due to lack of space) with different parameter settings and observed similar trends. The Kalman filter shows a slightly better performance than MMSE during the initial phase of spreading when the threshold level is small (say, $X_{L} \leq 0.15 N_{0}$ ). However, the Kalman filter outperforms the MMSE estimator for higher threshold levels (say, $X_{L} \geq 0.25 N_{0}$ ) because it takes into account all previous sample measures. 


\section{Conclusion}

In this paper, we tackled the problem of estimating file-spread in DTNs with two-hop routing. Apart from providing solid analytical basis to our estimation framework, we also provided insightful conclusions validated with simulations. Some of the important insights are: (i) the deterministic fluid model can indeed be a good predictor with a large number of nodes, (ii) the Kalman filter can track the spreading process quite accurately provided that measurements as well as updates are taken sufficiently fast, (iii) during the initial phase of spreading when the amount of sample measures is still low, the MMSE estimator can be used for estimating the level-crossing times of sufficiently low threshold levels, and (iv) as time progresses, the MMSE estimator becomes less useful, but the Kalman filter would be available at later phases to provide accurate estimates. Applying the real-time estimations for on-line adaptive control of the spreading process is a topic of our ongoing research.

\section{Appendix}

In this appendix, we provide a brief informal background on fluid and diffusion limits and approximations. Please refer to [6], 7] and [12 for more details.

Intuitively speaking, the fluid approximation provides the first-order deterministic approximation to a stochastic process and represents its average behavior. The diffusion approximation provides the second-order approximation to a stochastic process representing its average behavior added with random fluctuations about the average (usually, in terms of a Brownian motion).

Consider a sequence $\left\{Z^{(n)}(t), t \geq 0\right\}, n=1,2, \ldots$, of stochastic processes. Index $n$ represents some quantity which is scaled up to infinity in order to study the sequence of processes at the limit, as $n \uparrow \infty$. For queueing systems, $n$ might represent "the number of servers" (as in infinite server approximations) or "a multiplying factor of one or more transition rates" (as in heavy-traffic approximations) or some other quantity w.r.t. which the scaling is performed.

Consider the Strong Law of Large Numbers (SLLN) type rescaling $z^{(n)}(t):=$ $Z^{(n)}(t) / n$. Under certain conditions, as $n \uparrow \infty$, the sequence of rescaled processes $\left\{z^{(n)}(t), t \geq 0\right\}, n=1,2, \ldots$, converges almost surely to a deterministic process $\{z(t), t \geq 0\}$ (see, for example, Theorem 4.1 of [7]). Then, the limit $\{z(t), t \geq 0\}$ is called the fluid limit associated with the sequence $\left\{Z^{(n)}(t), t \geq 0\right\}, n=1,2, \ldots$, and the approximation

$$
Z^{(n)}(t) \approx n z(t), \quad \forall t \geq 0
$$

is called the fluid approximation for the $n$-th system.

Consider now the Central Limit Theorem (CLT) type rescaling $v_{z}^{(n)}(t)=$ $\sqrt{n}\left(z^{(n)}(t)-z(t)\right)$, which amplifies the deviation of the rescaled process $\left\{z^{(n)}(t), t \geq 0\right\}$ from the fluid limit $\{z(t), t \geq 0\}$. Under certain conditions, as $n \uparrow \infty$, the sequence of rescaled processes $\left\{v_{z}^{(n)}(t), t \geq 0\right\}, n=1,2, \ldots$, converges 
weakly to a diffusion process (or a continuous-time Markov process with continuous sample paths) $\left\{v_{z}(t), t \geq 0\right\}$ (see, for example, Theorem 4.2 of [7]). Then, $v_{z}(t)$ is called the diffusion limit associated with the sequence $\left\{Z^{(n)}(t), t \geq 0\right\}$, $n=1,2, \ldots$, and the approximation

$$
Z^{(n)}(t) \stackrel{d}{\approx} n z(t)+\sqrt{n} v_{z}(t), \quad \forall t \geq 0
$$

is called the diffusion approximation for the $n$-th system, where $\stackrel{d}{\approx}$ means "approximately distributed as". In particular, if $v_{z}(0)$ is a Gaussian random variable, then $\left\{v_{z}(t), t \geq 0\right\}$ is a Gaussian process and it is completely characterized by its mean and auto-covariance functions.

\section{References}

1. Alouf, S., Altman, E., Nain, P.: Optimal on-line estimation of the size of a dynamic multicast group. In: Infocom 2002, vol. 2, pp. 1109-1118 (June 2002)

2. Bremaud, P.: Point Processes and Queues: Martingale Dynamics. Springer (1981)

3. Karatzas, I., Shreve, S.E.: Brownian Motion and Stochastic Calculus. Springer, New York (1988)

4. Kurtz, T.G.: Solutions of Ordinary Differential Equations as Limits of Pure Jump Markov Processes. Journal of Applied Probability 7(1), 49-58 (1970)

5. Lindgren, A., Doria, A., Schelen, O.: Probabilistic Routing in Intermittently Connected Networks. In: ACM Mobicom (2003)

6. Mandelbaum, A., Massey, W., Reiman, M.I.: Strong approximations for markovian service networks. Queueing Systems 30, 149-201 (1998)

7. Mandelbaum, A., Pats, G.: State-dependent queues: approximations and applications. In: Kelly, F., Williams, R.J. (eds.) IMA Volumes in Mathematics and Its Applications, vol. 71, pp. 239-282. Springer, Berlin (1995)

8. Poor, H.V.: An Introduction to Signal Detection and Estimation, 2nd edn. Springer (1994)

9. Simon, D.: Optimal State Estimation. John Wiley \& Sons, Inc. (2006)

10. Spyropoulos, T., Psounis, K., Raghavendra, C.: Efficient Routing in Intermittently Connected Mobile Networks: The Multi-copy Case. IEEE/ACM Transaction on Networking 16(1), 63-76 (2008)

11. Vahdat, A., Becker, D.: Epidemic Routing for Partially-Connected Ad Hoc Networks. Duke University, Tech. Rep. CS-200006 (2000)

12. Whitt, W.: Stochastic-Process Limits. Springer (2002)

13. Zhang, X., Neglia, G., Kurose, J., Towsley, D.: Performance Modeling of Epidemic Routing. Computer Networks 51, 2867-2891 (2007) 